\title{
Health Reference Information Model Architecture Research Issues and Challenges
}

\author{
Samir K Amin \\ Faculty of Computing and IT, \\ Sohar University, Oman
}

\author{
Dinesh Kumar Saini \\ Faculty of Computing and IT, \\ Sohar University, Oman \\ Adjunct Faculty of Engineering \\ and IT, \\ University of Queensland, \\ Brisbane, Australia
}

\author{
Hemant Gaur \\ M.Tech. (CS) Student \\ Department of Computer \\ Science and IT \\ Bhagwant University \\ Ajmer Rajasthan India
}

\begin{abstract}
The purposed Customization of patients care history in Personal health record (PHR) in hospital information system is to improve their health care service, about to build a database for the patient history in the hospital. It is very much needed in the current times because hospitals get many patients from various referral sources and most of the time the new hospital don't have patient history with them. All the tests and analysis carried out on the patients are asked to get it done again which cost lot of money and time. This kind of problem can be solved by adopting the Health Information Reference Model and adopting the HIS-HL-7. Standards are developed; the question is how to adopt them in different countries and different hospitals. If there is a framework and model that can help them to adopt this, it could prove to be very useful. In this paper efforts are made to analyses and study the health information reference model. Data quality, cost and patient care are the main areas of concerns for almost all health information models [1].
\end{abstract}

\section{General Terms}

Software Systems, Computing, Health Systems, Models, Research, Future Directions.

\section{Keywords}

Health Reference Information Model, HIS-HL-7 Model, Research Issues

\section{INTRODUCTION}

Most of the developing countries are building their health infrastructure and it is very premature stages. It is suggested that these developing infrastructure countries can adapt the existing models which are at least well researched; even these models also have issues that need to be addressed[2,3,4,5]. Authors suggest that HL7 can be adapted in the developing countries. Heath Level Seven (HL7) is an ANSI-accredited Standards Developing Organization (SDO) operating in healthcare arena; it provides standards for data exchange to allow interoperability between healthcare information systems. HL7 focuses on the clinical and administrative data domains $[6,7,8]$. This is a not-for-profit organization made up of volunteers - providers, payers, vendors, government, and key goal is syntactic and semantic interoperability $[9,10,11]$.

\subsection{Benefits of using HL7}

Recommended by the Health and Human Service (HHS) as the messaging standard for electronic exchange of clinical data, and this can be directly adapted in the developing countries $[12,13,14]$.
Over $90 \%$ of US hospitals have implemented some version of 2.x HL7 messages.

Support for translational research requires integration of research data with the clinical data.

$\mathrm{V} 3$ offers the syntactic and semantic interoperability to make this possible

\subsection{Structure of HL7}

There are 4 main pillars of semantic interoperability and these are as follow:

$>$ A common Reference Information Model (RIM) spanning the entire clinical, administrative and financial healthcare universe

> A well-defined and tool-supported process for deriving data exchange specifications ("messages") from the RIM

$>$ A formal and robust Data Type Specification upon which to ground the RIM

$>$ A formal methodology for binding concept-based terminologies to RIM attributes.

\subsubsection{Reference Information Model (RIM)}

Follows object oriented developmental methodology based on a UML model, it represents the fundamental model from which all v3 messages are derived is referred to as the Reference Information Model (RIM), it is a generic, abstract model that expresses the information content of all the areas of healthcare [16,17]. It defines all the information from which the data content of HL7 messages are drawn and it forms a shared view of the healthcare domain and is used across all HL7 messages independent of message structure.

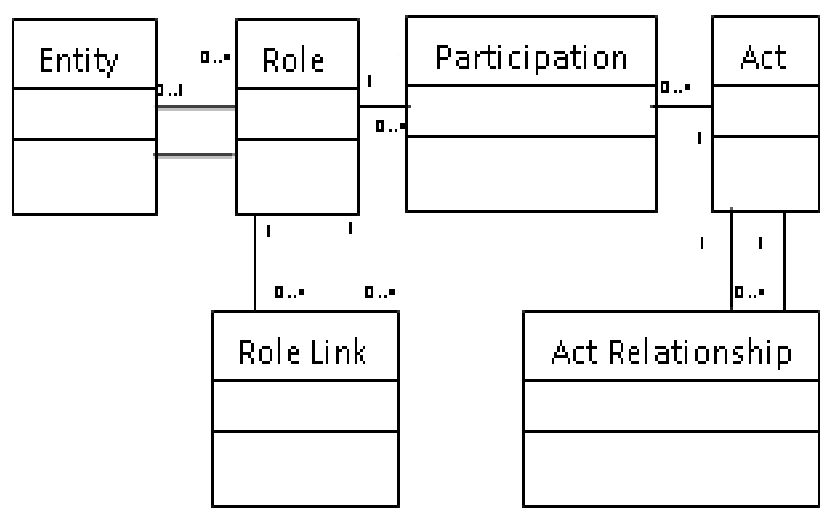

Figure.1 Reference Information Model 


\section{THE CLASSES IN THE MODEL}

\subsection{Entity class}

This class represents a person, animal, organization or thing, it contain a collection of classes representing specializations and related qualifying classes. The classes represent health care stakeholders and other things of interest to health care, it has the following sub-classes :

Container, Device, LanguageCommunication, LivingSubject, ManufacturedMaterial, Material, NonPersonLivingSubject, Organization, Person and Place. The super class has the following attributes, each attribute can be inherited by a sub class, these attributes are:

classCode, determinerCode, id, code, quantity, name, desc, statusCode, existenceTime, telecom, riskCode, handlingCode.

\subsection{Role class}

Represents responsibility or part played by an entity (e.g. Person in a role of patient, employee, etc.) - a collection of classes related to this class and its specializations can represents different faces of an Entity. These classes focus on the roles participants may play in health care. Role class has the following attributes:

classCode, id, code, negationInd, addr, telecom, statusCode, effectiveTime, certificateText, quantity, positionNumber. Role has the following sub-classes:

Access, Employee, LicensedEntity and Patient.

\subsection{Participation class:}

This is an association class between an Act class and a Role class with an Entity playing that Role. Participation has the following sub-class (ManagedParticipation). The Participation class attributes are:

typeCode, functionCode, contexCotrolCode, sequenceNumber, negationInd, noteText, time, modeCode, awarenessCode, signatureCode, signatureText, performInd, substitutionConditionCode.

\subsection{Act class}

This class represents a record of something that is being done, has been done, can be done, or is intended or requested to be done. A collection of classes including the Act class and its specializations will relate to the actions and events that constitute health care services.

\subsubsection{Act class has the following sub-classes}

Account, ControlAct, DeviceTask, DiagnosticImage, Diet, FinancialContract, FinancialTransaction, InvoiceElement, Observation, Participation, PatientEncounter. Procedure, PublicHealthCase, SubstanceAdministration, Supply and WorkingList.

\subsubsection{Act class attributes are as following:}

ClassCode, moodCode, id, code, negqtionInd, derivationExpr, text, title, statuseCode, effictiveTime, activityTime, availabilityTime, priorityCode, confidentialityCode, repeatNumber, interruptibleInd, levelCode, independentInd, uncertaintyCode, reasonCode, languageCode.

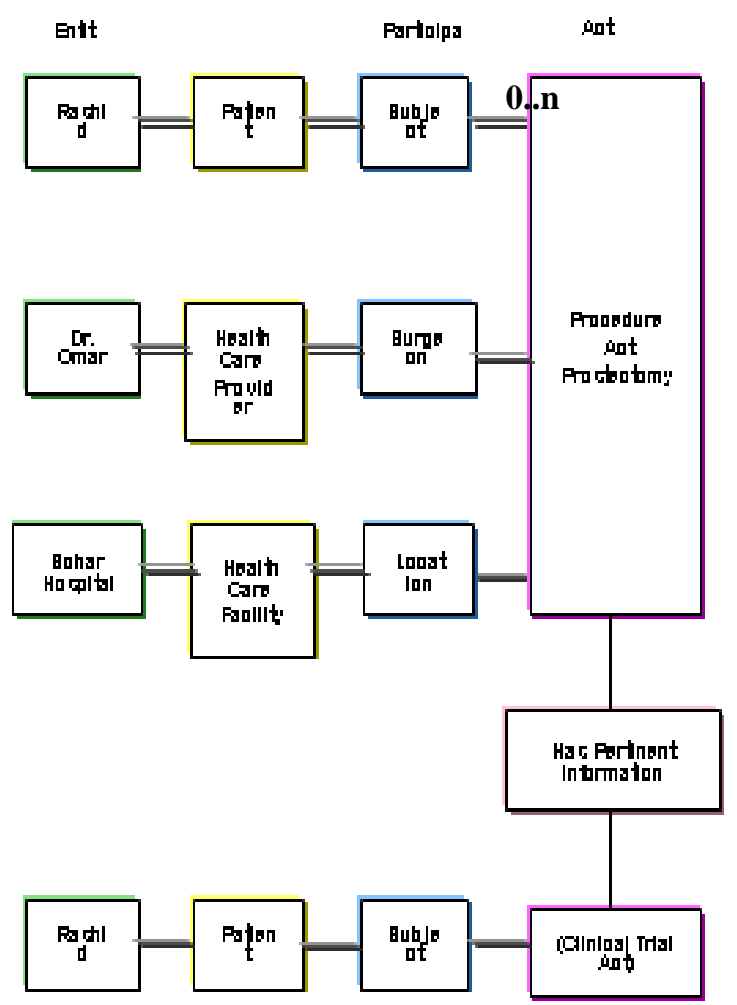

Figure2. Instance Scenario of RIM

The need of internationally acceptable standards to ensure systematic electronic data interchange (EDI) in the health care field led to development of Health level 7 in 1987[3].

HL7 has seven layers of interoperability namely the Testing Business Process, Application, Transport and Network Layers [4]. In the US, most of the electronic health data is already transferred to this standard $[5,6]$.

-Data transfer via networks - The computer network systems must be compatible in order to allow standard-based communication among various kinds of healthcare applications [7].

-Error in Data - HL7 messages use simple delimiters to separate data, this makes the message difficult to decipher and is easily error-prone [3]. There are projects that are exploring the error in message parsing and it help

\section{INTEROPERABILITY AND TESTING OF HL7}

The ability of systems to exchange and use processed data is called interoperability. Interoperability is also called the ability of systems to exchange data in such a manner that the system receiving the processed data or service request can perform the task efficiently without the support from another secondary operator.[22,23,25] 


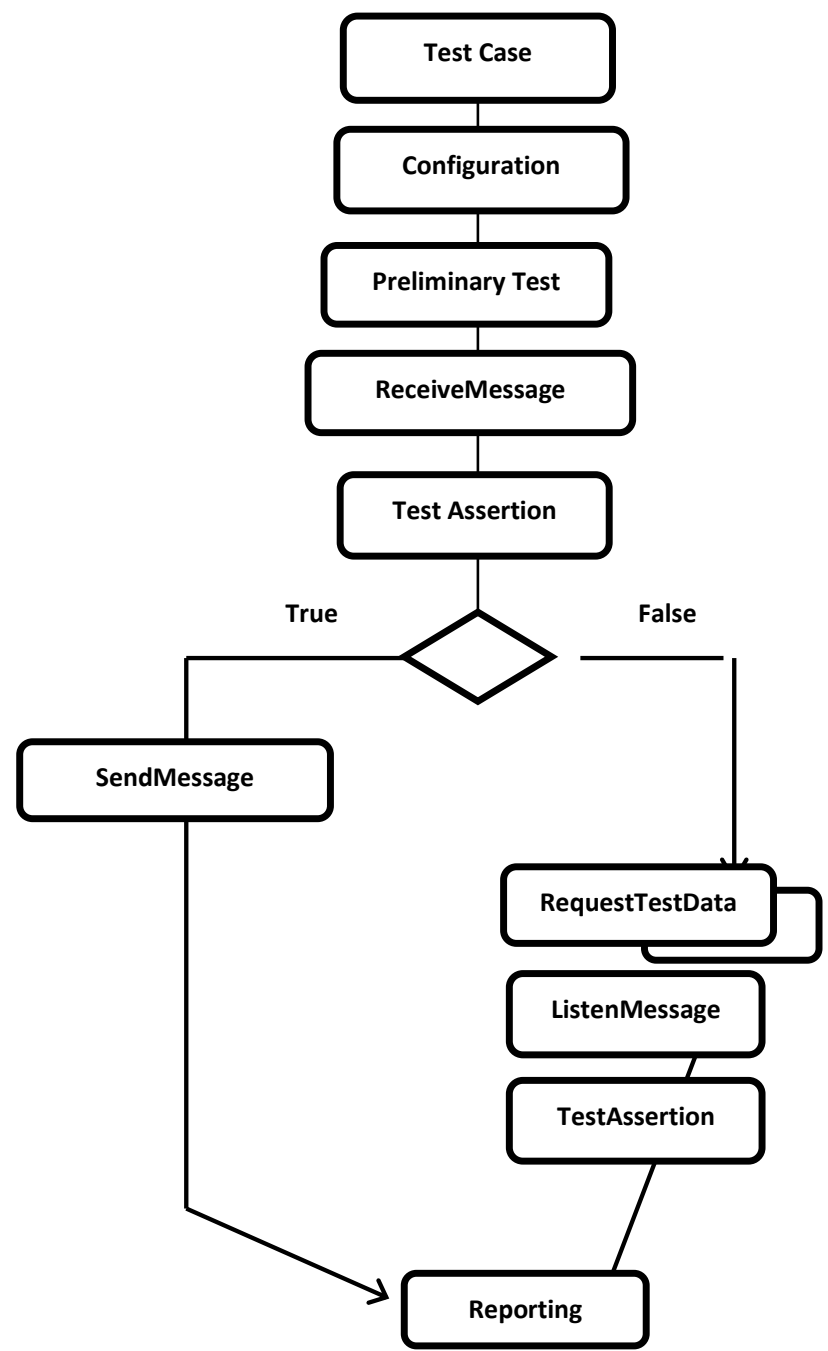

Figure 1 - Flow of Testing in HL-7

\section{HL7 VERSION 3 AT NCICB}

Highlights of the NCICB Clinical Architecture Vision

-caAdapter - Open source toolkit which facilitates v3 message building, parsing and validation, caAdapter Facilitates the building, parsing, and validation of HL7 v3 messages

-Message Exchange - Service supporting message transmission and routing

-HL7 Transactional Database - HL7 RIM-based transactional database and data access service
-De-identification Service - Service for de-identifying patient information from HL7 messages

-Analytical Applications - Applications for querying, reporting, mining clinical data

-Cancer Data Standards Repository (caDSR) - Shared metadata repository

-Enterprise Terminology Services (EVS) - Terminology service for hosting controlled vocabulary, including HL7 registered terminology

\subsection{Components Include}

HL7 v3 Message Parser - parses HL7 version 3 messages to RIM object graph

HL7 v3 Message Builder - builds HL7 version 3 messages from the RIM object graph

HL7 v3 Artifacts - implements RIM objects, data types and metadata objects

Metadata Loader - represents HL7 version 3 metadata inmemory

Validation Services - integrates with NCICB caCORE components such as Enterprise Vocabulary Service (EVS) and W3C XML schema validation services

Mapping Tool - maps clinical data to the HL7 version 3 message specification currently, with extensible mapping and data transformation in the near future

caAdapter API - uses mappings to generate HL7 version 3 messages

Message Service - integrates with message exchange services (future plans)

A platform to enable clinical applications to build and parse HL7 v3 messages based on specific schema definitions $[20,21,22]$.

Capability to perform vocabulary validation of the RIM structural attributes.

An open source solution for implementing an international messaging standard allowing data to be exchanged between disparate systems.

Integration with NCI metadata repository (caDSR) and terminology services (EVS)

Facilitating the building and parsing of HL7 v3 messages from source clinical systems will promote data exchange in a standards based common format. 


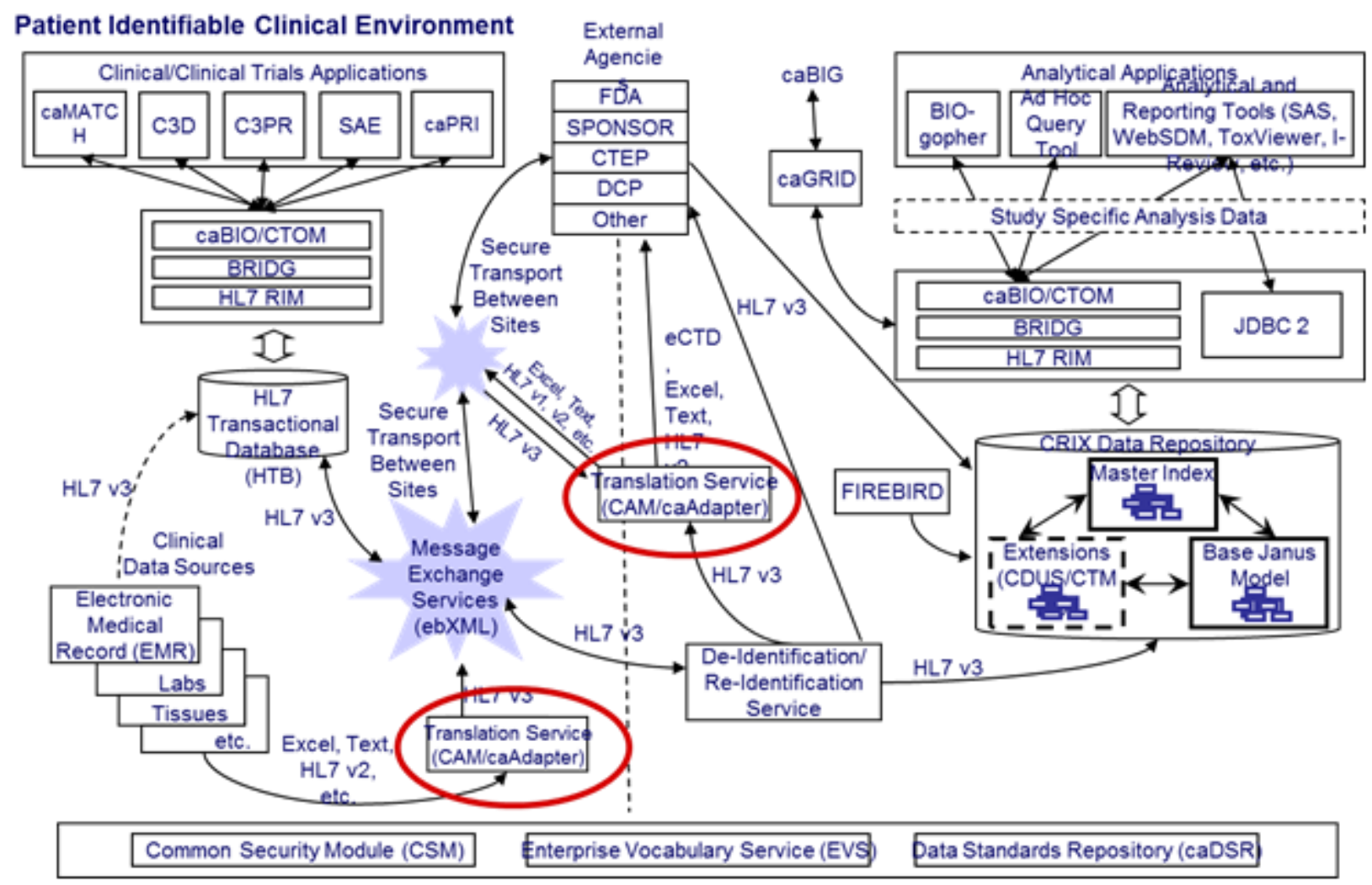

Figure3: Clinical Environment for Patient Record managed in HIS systems using HL-7 v3.

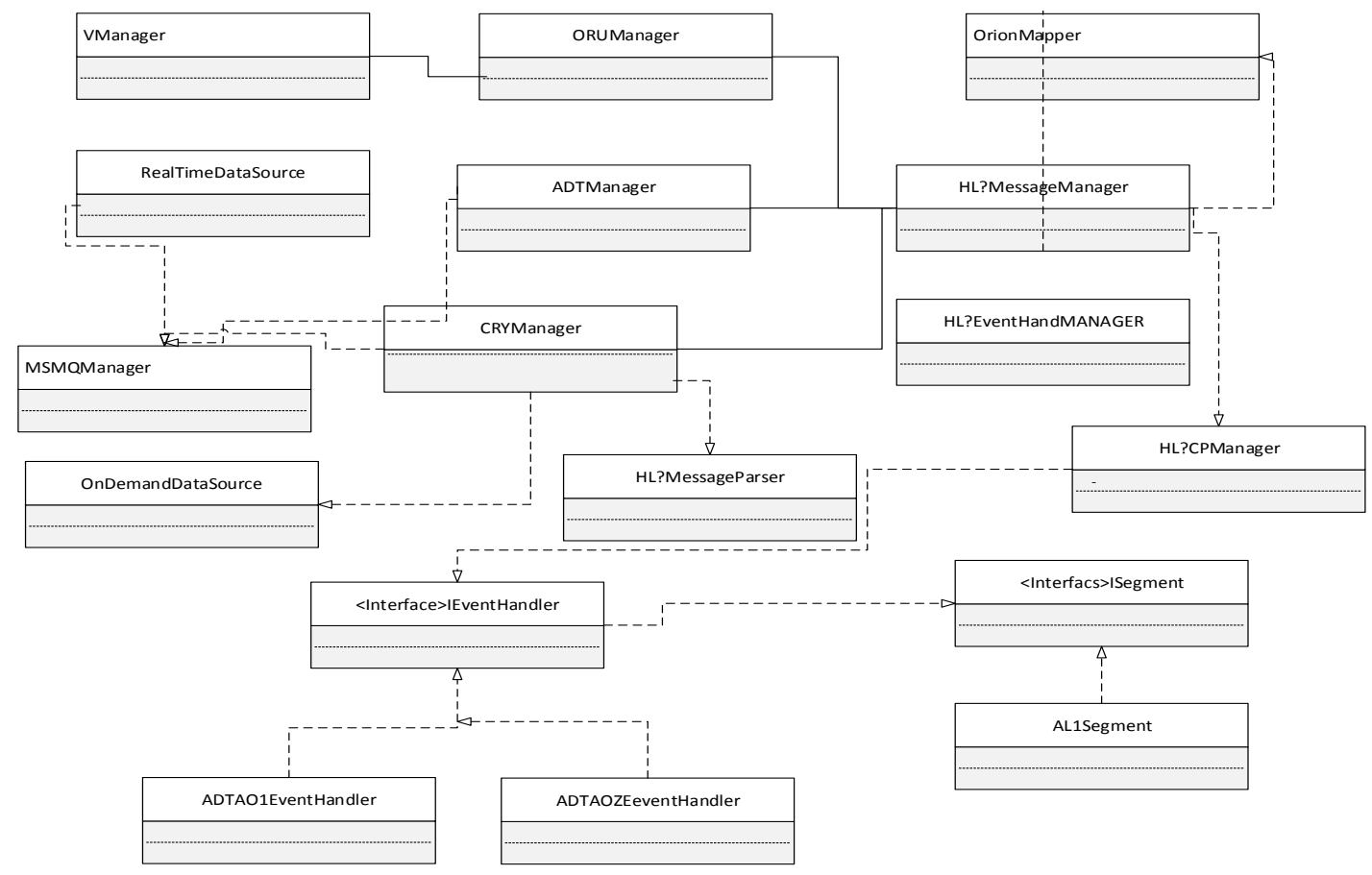

Figure4.Class Diagram for HIS-HL7

The QRYManager feeds into OnDemandDataSource which runs four requests GetData, GetMessagebyPatient, GetMessagebyUnit, GetMessagebyFacilitiesList, GetMessagesByParameter.sends messge to MSMQManager and HL7MessageParser.

The HL7TCPManager sends message QRYManager
The IEventHandler interface has the following methods AddRequiredSegments,

PrepareMessage,AddOptionalSegments

There are two classes ADT01EventHandlers and ADT02EventHandler that inherit from the IEventHandler class. 
The AL1Segment class inherits interface ISegment and this class has method AddSegmentData (in MessageData).

\section{CONCLUSION}

In this paper we emphasize the importance of the standards and how these standards help in improving the patient care. Model and adopting the HIS-HL-7. Standards are developed; the question is how to adopt them in different countries and different hospitals. If there is a framework and model that can help them to adopt this, it could prove to be very useful. In this paper efforts are made to analyses and study the health information reference model. Data quality, cost and patient care are the main areas of concerns for almost all health information models. There is a scope for health sector for multinational research participation in this area.

\section{ACKNOWLEDGEMENT}

Author would like to thank Faculty of Computing and Information Technology, Sohar University and Faculty of Engineering and Information Technology University of Queensland Brisbane, Australia, for supporting and providing research opportunity. The author would also like to thank the reviewers for the review and the input provided for the paper.

\section{REFERENCES}

[1] Dinesh Kumar Saini "Sense the Future" Campus Volume 1- Issue 11, Page No14-17, February 2011.

[2] Hutchison, A., et al., Electronic data interchange for healthcare. IEEE Comput.Mag, 1996. 34: p. 28-34.

[3] M. Dixon, S.C., B. Read, . , Implications of WWW technologiesfor exchanging medical records. J. Inform Prim. Care, 1999: p. 3-9.3. Huang, E.W., S.H. Hsiao, and D.M. Liou, Design and implementation of a web-based HL7 message generation and validation system. International journal of medical informatics, 2003. 70(1): p. 49-58.

[4] [Online]. Event-Driven Test Scripting Language (eTSL). OASISebXML Implementation Interoperability and Conformance (IIC) TC,Working Draft 0.85 . 2007.

[5] J.D. Halamka, C.O., C. Safran,,, CareWeb, a webbasedmedical record for an integrated healthcare deliverysystem. Int. J. Med. Inf. 54

[6] D.S. Greenberg, B.W., Int. J. Med. Inf. 48 ActiveXbased standards for healthcare integration: p. 183/190.

[7] J. Dudeck, Aspects of implementing and harmonizinghealthcare communication standards. Int. J. Med. Inf., 1998. 48: p. 163/171.8. Namli, T., G. Aluc, and A. Dogac, An interoperability test framework for HL7-based systems. Information Technology in Biomedicine, IEEE Transactions on, 2009. 13(3): p. 389399.

[8] www.hl7.org

[9] https://www.hl7.org/library/datamodel/RIM/C30202/rim.htm

[10] http://www.hl7.org/library/datamodel/RIM/C30123/vocabulary.htm

[11] HL7 Version 3 Standard, http://www.hl7.org/v3ballot/html/welcome/environment/ index.htm

[12] NCICB caAdapter Project Site, trials.nci.nih.gov/projects/infrastructureProject/caAdapter
[13] HL7 Version 3: Driving Interoperability \& Transforming Healthcare Information Management - Charles Mead, $\mathrm{MD}, \mathrm{MSc}$

[14] NIHSeminar_Charlie Mead.ppt

[15] Dinesh Kumar Saini and Bimal Kumar Mishra "Design Patterns and their effect on Software Quality" Vol.5, No.1, January 2007, Page356-365 ACCST Research Journal, INDIA

[16] Dinesh Kumar Saini and Hemraj Saini "Proactive Cyber Defense and Reconfigurable Framework for Cyber Security" International Review on computer and Software (IRCOS) Vol.2. No.2. March 2007, 89-98. ITALY

[17] Dinesh Kumar Saini and Nirmal Gupta "Fault Detection Effectiveness in GUI Components of Java Environment through Smoke Test", Journal of Information Technology, ISSN 0973-2896 Vol.3, issue3, 7-17 September 2007.

[18] Dinesh Kumar Saini and Nirmal Gupta "Class Level Test Case Generation in Object Oriented Software Testing, International Journal of Information Technology and Web Engineering, (IJITWE) Vol. 3, Issue 2, pp. 19-26 pages, march 2008. USA

[19] Dinesh Kumar Saini "Testing Polymorphism in Object Oriented Systems for improving software Quality” ACM SIGSOFT Volume 34 Number 2 March 2009, ISSN: 0163-5948, USA

[20] Lakshmi Sunil Prakash, Dinesh Kumar Saini and Kutti N.S. "Integrating EduLearn Learning Content Management System (LCMS) with Cooperating Learning Object Repositories (LORs) in a Peer to Peer (P2P) architectural Framework" ACM SIGSOFT Volume 34 Number 3 May 2009, ISSN: 0163-5948, USA.

[21] Dinesh Kumar Saini, Jabar H. Yousif, and Wail M. Omar "Enhanced Inquiry Method for Malicious Object Identification" ACM SIGSOFT Volume 34 Number 3 May 2009, ISSN: 0163-5948, USA.

[22] H Saini, D.K.Saini and N.Gupta "E-Business System Development: Review on Methods, Design Factors, Techniques and Tools with an Extensive Case Study for Secure Online Retail Selling Industry" Journal of science and Technology (ISSN 0974-6846), Vol.2. No.5, May 2009, India.

[23] Wail M.Omar, Dinesh K. Saini and Mustafa Hassan "Credibility Of Digital Content in a Healthcare Collaborative Community" Software Tools and Algorithms for Biological Systems in book series "Advances in Experimental Medicine and Biology, AEMB" Springer, Page No, 2010

[24] Dinesh Kumar Saini, Sanad Al Maskari and Lingraj Hadimani " Mathematical Modeling of Software Reusability" 3rd IEEE International Conference on Machine Learning and Computing (ICMLC 2011)Singapore, February 26-28, 2011, IEEEXplore, and indexed by INSPEC, Ei Compendex and ISI Proceedings.

[25] Dinesh Kumar Saini, Wail M Omar and Sanad Al Maskari "Healthcare Collaborative Framework for Chronic Disease Management in Oman" proceedings of 
International Conference on Multidisciplinary Approaches to Diabetes Research and Health (ICMADRH-2010), India, pp 46-52.

[26] Dinesh K Saini, Mustafa Hasan "Architecture and Classification algorithms for large P2P Digital Library" The Second International Conference on Networked Digital Technologies OpenConf system, Crez Republic Europe 2010.

[27] Dinesh Kumar Saini, Wail M. Omar "Software Testing For Semantic Service Oriented Architecture for E-Health Software Services" SERP'10 - 9th international Conference on Software Engineering Research and Practice (USA) http://www.world-academy-ofscience.org/, P.No. 240-246.

[28] Dinesh Kumar Saini and Mustafa Hasan "Building Digital Library for Large P2P Networks" IKE'10 - 9th International Conference on Information and Knowledge Engineering (2010, USA) http://www.world-academyof-science.org/ P.No. 185-189

[29] Dinesh Kumar Saini, Wail M. Omar "Web 2.0 and Healthcare collaborative Framework for Health Awareness and Education" BIOCOMP'10 - 11th International Conference on Bioinformatics and Computational Biology (2010, USA) http://www.world-academy-of-science.org/ p. no 561564

[30] Osama A Rahmeh, Princy Johnson and Dinesh Kumar Saini "A Latency Optimizing Load Distribution Scheme for Grid Networks" 8th international conference on Grid Computing and Application (GCA2101), July 12-15, 2010, USA, http://www.world-academy-ofscience.orgP.No. 70-76.

[31] Dinesh Kumar Saini, Osama Abu Rahmeh, H. Saini, Wail M. Omar "Extended Secure Architecture of HIS: HL7" BIOCOMP'10 - 11th International Conference on Bioinformatics and Computational Biology (July 12-15, 2010, USA) http://www.world-academy-of-science.org, P.No. 617-623.

[32] Dinesh K Saini, Wail M Omar , Mustafa Hassan, Khamsa Enaya and Saima Yazdani "University- Industry Linkage: Sohar University Experience with Case Study on Cisco Event as Experience for Learning in Developing Local Talent in Sultanate of Oman" MIT LNC Conference 2010 May 23-26 MIT USA .

[33] Dinesh Kumar Saini, Lakshmi Sunil Prakash and Wail M Omar "Review of Technological Challenges in Web Based Learning Content Management Systems (LCMS) with special emphasis on extraction of Learning Contents" International Symposium, College of Applied Science, Ministry of Higher Education, April 13-16, 2010, Oman, P.No. 43-49.

[34] Dinesh Kumar Saini and Hemraj Saini "Cyber Defense: Use of AI Techniques," 3rd International Conference on Quality, Reliability and INFOCOM Technology (Trends and Future Directions), 2-4 December, 2006, Indian National Sciences and Academics, New Delhi (India). Conference proceeding.
[35] Dinesh Kumar Saini and Hemraj Saini "Cyber Defense Architecture in Campus Wide Network," 3rd International Conference on Quality, Reliability and INFOCOM Technology (Trends and Future Directions), 2-4 December, 2006, Indian National Sciences and Academics, New Delhi (India) conference proceeding.

[36] Dinesh Kumar Saini and Hemraj Saini "Achieving Quality Through Testing Polymorphism in Object Oriented Systems,"3rd International Conference on Quality, Reliability and INFOCOM Technology (Trends and Future Directions), 2-4 December, 2006, Indian National Sciences and Academics, New Delhi (India). Conference proceeding.

[37] Dinesh Kumar Saini and Hemraj Saini "Issues and Problems in Generation of Automated Test Data for Object Oriented Systems," 3rd International Conference on Quality, Reliability and INFOCOM Technology (Trends and Future Directions), 2-4 December, 2006, Indian National Sciences and Academics, New Delhi (India) Conference proceeding.

[38] Dinesh Kumar Saini and Hemraj Saini "Statistical Modeling of Extensibility in software" 3rd International Conference on Quality, Reliability and INFOCOM Technology (Trends and Future Directions), 2-4 December, 2006, Indian National Sciences and Academics, New Delhi (India). ISBN 81-7446-434-4 Conference proceeding.

[39] Dinesh Kumar Saini and Hemraj Saini "Compliance Management Framework and Methodology", 8th International Research Conference on Quality, Innovation and Knowledge Management, Feb 11-14, 2007 at IMT New Delhi Sponsored by CII, IBEF, Monash University .

[40] Dinesh Kumar Saini and Hemraj Saini "An Exploratory Study of Software Metrics on Performance Issues Of Web Services" International Conference on Controls, Automation and Communication Systems (ICCACS) at Bhubneswer December 2004.

[41] Dinesh Kumar Saini and Hemraj Saini "Quality and Reliability of Bluetooth Technology" international Conference on Quality Reliability and Control (In Communication and Information Systems) I.I.T.Mumbai, , ISSN 0972-6454, December 2001.

[42] Hemraj Saini and Dinesh Kumar Saini "AN Automated Test framework for Java Application" ICIT (IEEE Sponsored) Rourkella-2007.

[43] Dinesh Kumar Saini and Hemraj Saini "Software Metrics and Mathematical Models in the Software Development Environment for Improving its Quality", National Conference on Mathematical Modeling, BITS Pilani. Oct.2005.

[44] Dinesh Kumar Saini and Hemraj Saini "Analytical Study of Mathematical Models For Software Reusability Metrics in Software Development Environment" National Conference on Mathematical Modeling and Analysis - October 2004 